\title{
HYALURONIDASE IN OCULAR TISSUES* \\ I. SENSITIVE BIOLOGICAL ASSAY FOR SMALL CONCENTRATIONS OF HYALURONIDASE
}

\author{
BY \\ G. MAYER \\ Haifa
}

SEVERAL methods for the estimation of hyaluronidase have been described:

(1) The spreading reaction is a biological method, measuring the decomposing effect of hyaluronidase on the mucoids of the cement substance of rabbit's skin (Duran-Reynals, 1929). These substances consist partly of hyaluronic acid. Hyaluronidase increases the permeability of the tissues, and this involves an increased spreading capacity of liquids and corpuscular substances.

This test is not specific for hyaluronidase as some unspecific agents also give a spreading reaction.

(2) The viscosity-reducing method is based on the fact that hyaluronic acid is a substance of high viscosity (Madinaveitia and Quibell, 1940). The reduction of the viscosity caused by hyaluronidase is measured by this method. "

(3) The turbidimetric method is most extensively used (Kass and Seastone,1944). It is based on the observation that pure hyaluronate at $p \mathrm{H} 4 \cdot 2$ forms a stable turbid colloidal suspension with dilute serum, whereas depolymerized hyaluronate remains clear in these conditions.

(4) Mucin clot prevention depends on the observation that native hyaluronic acid in acid solution coprecipitates with protein in a fibrous " mucin" clot (Robertson, Ropes, and Bauer, 1940). After incubation with hyaluronidase, hyaluronic acid becomes depolymerized and the mucin clot formation is prevented. The mechanism of this test is not clear.

(5) The chemical method consists in measuring the increase in reducing sugar, or in liberated acetyl-glucosamine after depolymerization of hyaluronic acid.

None of the methods mentioned above is sensitive enough for the determination of small quantities of hyaluronidase, for example those possibly present in the tissues of the eyes.

The bacteriological decapsulation method is based on the observation that certain strains of mucoid streptococci of the Lancefield Groups A and C under definite conditions produce a capsule consisting of hyaluronic acid, which is dissolved in the presence of hyaluronidase (McClean, 1941a,b, 1942; Hirst, 1941).

The bacteriological method is the most sensitive one but has been objected to (Meyer, 1947) because these organisms may lose their capsules under various conditions without added enzyme.

* Received for publication June 14, 1955. 
Murray and Pearce (1949) developed a bacteriological method based upon the characteristics of certain mucoid strains of Streptococcus pyogenes growing on agar. These colony characteristics were specifically changed in the presence of hyaluronidase, and hyaluronidase activity could thus be demonstrated.

Repeated use of the method of Murray and Pearce gave very variable results in this laboratory.

By using in a different way the principle of decapsulation a sensitive bacteriological method has been developed which has enabled us to estimate hyaluronidase in very small concentrations.

\section{Materials}

The medium used for maintaining cultures and for the test was brain heart infusion (Baltimore Biological Laboratory) $p \mathrm{H} \mathrm{7.4}$ with the addition of 1.2 per cent. of granulated agar.

The blood had to be added while the medium was at $60^{\circ}-65^{\circ} \mathrm{C}$. Each ampoule of hyaluronidase contained 25 Cilag viscosity reducing units (V.R.U.) which correspond roughly to 75 turbidity reducing units (T.R.U.).

A Lancefield Group A Type 9 Streptococcus pyogenes (“ M Str. 9 ”), kindly supplied for this purpose by Dr. R. G. E. Murray, was used as substrate organism.

To keep the organism in its mucoid and capsulated state the following method gave satisfactory results:

The streptococcus was incubated in the liquid medium for $2-5 \mathrm{hrs}$; it was then transferred to a solid slant, from which it was again transferred after 16-18 hrs' incubation to the liquid medium. It was therefore necessary to transfer the strain twice daily. A stock of the capsulated organism was kept in a lyophilized state.

Capsule staining from the liquid medium was made by an Indian-ink-carbol fuchsin method.

\section{METHOD}

The streptococci were incubated from the 16- to 18-hours-old solid-medium culture, into three test tubes with the liquid medium. This was incubated for $2 \mathrm{hrs}-$ after which time capsule staining was performed from each tube. From the beginning of this incubation, the duration of the experiment should not exceed 5 hours, after which time spontaneous decapsulation might occur.

The culture showing the largest mean size of capsules was used for the experiment. The contents of this tube were distributed into Widal test tubes, $0.5 \mathrm{ml}$. being inserted into each one.

A series of dilutions of hyaluronidase in saline was prepared. From this series $0 \cdot 2 \mathrm{ml}$. of each dilution was added to each of the tubes containing the culture. Three tubes were set up as controls, e.g. $0 \cdot 2 \mathrm{ml}$. saline was added to each of them instead of hyaluronidase.

All the tubes were now incubated for 2 hours. After this period capsule stainings were performed - two slides from each tube. Fifty chains were examined on each slide-altogether a hundred chains from every dilution. The chains were divided into two groups:

Group 1.-Chains without or with very small capsules;

Group 2.--Chains with medium and with big capsules (Figs 1, 2, and 3, opposite).

*The hyaluronidase used during these investigations was produced by " Cilag" Limited, Schaffhausen, Switzerland. 


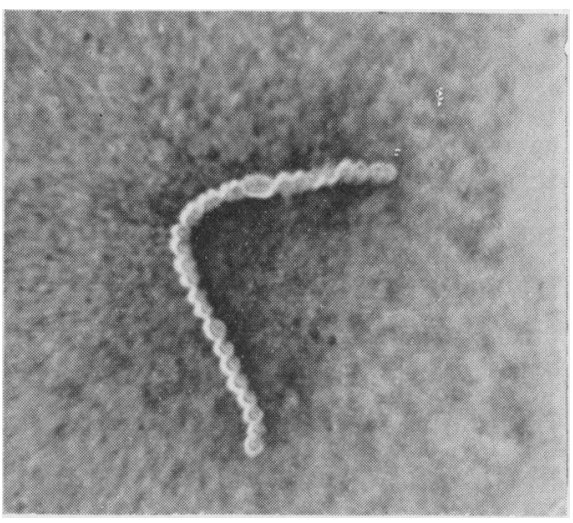

Fig. 1.-Small capsule.

\section{Results}

The results were evaluated bycalculating the percentage of streptococci without or with very small capsules in each dilution.

Fig. 4 shows that the series of dilutions of hyaluronidase caused decapsulation of 100 per cent. of the streptococci down to the concentration of 0.025 T.R.U. per ml., and 96 per cent. of the streptococci down to the concentration of 0.0125 T.R.U. per $\mathrm{ml} . \quad 70$ per cent. of streptococci became decapsulated by the concentration of 0.006 T.R.U. per ml.

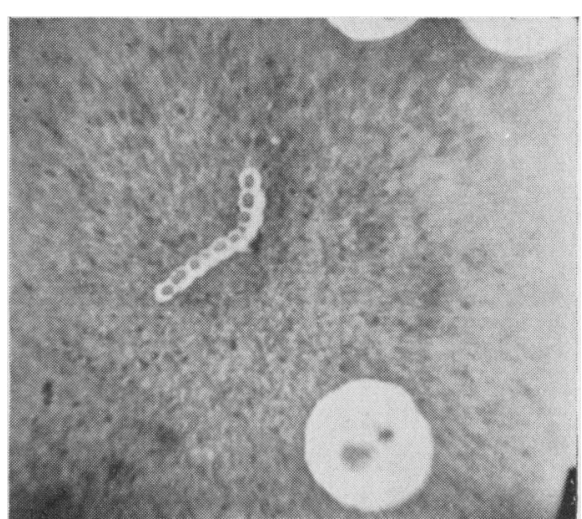

FIG. 2.-Medium capsule.

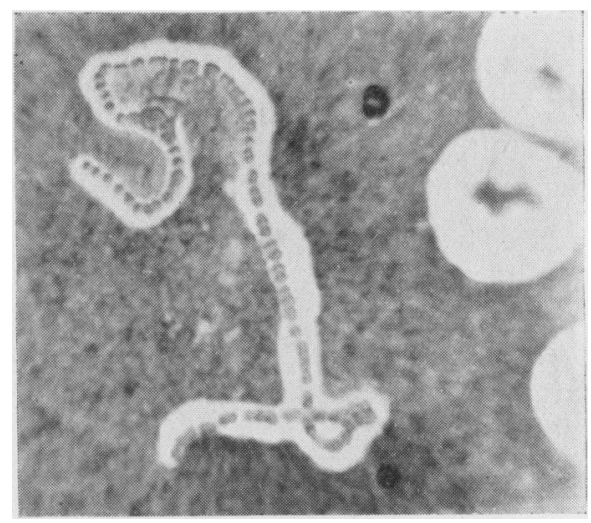

Fig. 3.-Big capsule.

The controls contained 15,10 , and 7 per cent. of chains without capsules or with very small capsules.

Fig. 5 demonstrates a similar experiment with a series of dilutions of

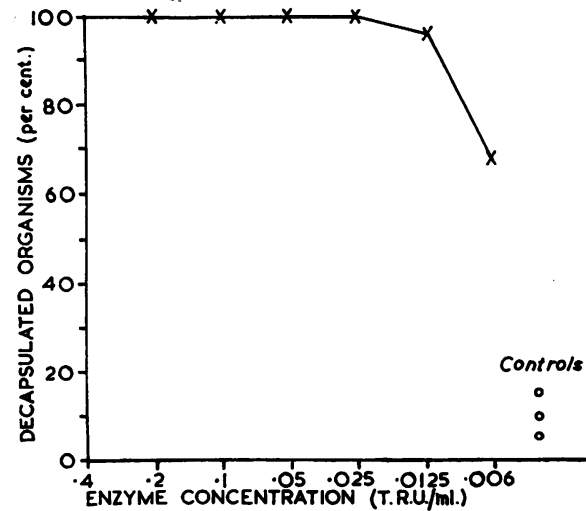

Fig. 4.-Relationship of concentrations of hyaluronidase to percentage of decapsulated organisms, with three controls.

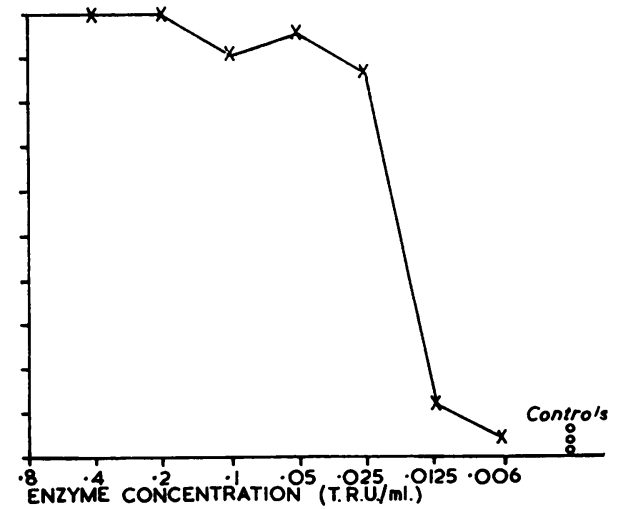

FIG 5.-Relationship of concentrations of hyaluronidase to percentage of decapsulated organisms, with three controls. 
hyaluronidase. Concentrations of hyaluronidase approximating to only 0.025 T.R.U. per ml. caused decapsulation of 90 per cent. of the streptococci, whereas 0.0125 T.R.U. per ml. caused no decapsulation.

The concentration of 0.0125 T.R.U. per ml. came within the range of the controls without hyaluronidase.

The further experiments were made at the same time with the same dilutions of hyaluronidase using the same streptococcus culture. The results were similar: 0.025 T.R.U. per ml. caused decapsulation.

In order to estimate the sensitivity of the test with tissue extracts we examined tissue from rabbits' testes. 1.3' gr. testes were mashed in a glass homogenizer. The dilution of $1: 780 \cdot 000$ per gr. testes gave evidence of the presence of hyaluronidase activity. This experiment proved again the sensitivity of the method used.

\section{Discussion}

Bacteriological decapsulation appears to be the most sensitive method for the estimation of hyaluronidase. The disadvantage of this method was the possibility of spontaneous decapsulation without added hyaluronidase. To avoid unspecific decapsulation the following measures were taken:

(1) The organism was maintained in a fairly stable mucoid state using the devices described above.

(2) One out of three 2-hour-old cultures were chosen, having been proved by preceding examination to be most suitable for the experiment because it had the largest mean size of capsules and the least variation in capsule size among the individual chains.

(3) The time of the entire experiment was kept within the limits of 5 hours, after which time spontaneous decapsulation often appears.

\section{Summary}

A sensitive bacteriological method of assay for hyaluronidase is described. This method enables the detection of small concentrations of the enzyme down to a limit of between 0.025 to 0.0125 T.R.U. per ml.

This work was carried out in the ophthalmological laboratory of the Rambam Government Hospital, Haifa, under the direction of Dr. I. C. Michaelson. The work was supported by a grant from the Arnold Reuben Fight for Sight Fund of the National Council to Combat Blindness Inc., New York.

The author is indebted to Dr. I. C. Michaelson for his continuous help and advice, and to Miss Eva Lewkowitz for her technical assistance.

\section{REFERENCES}

Duran-Reynals, F. J. (1929). J. exp. Med., 50, 327.

HIRST, G. K. (1941). Ibid., 73, 493.

Kass, E. H., and SEASTONE C. V. (1944). Ibid., 79, 319.

Madinaveitia, J., and Quibell, T. H. H. (1940). Biochem. J., 34, 625.

MCClean, D. (1941a). J. Path. Bact., 53, 13.

(1941b). Ibid., 53, 156.

(1942). Ibid., 54, 284.

MEYER, K. (1947). Physiol. Rev., 27, 335.

Murray, R. G. E., and PeARCE, R. H. (1949). Canad. J. Res., Sect. E., 27, 254.

Robertson, W. van B., Ropes, M. W., and BAuer, W. (1940). J. biol. Chem., 133, 261. 